\title{
Sublimation rate of ice under simulated Mars conditions and the effect of layers of mock regolith JSC Mars-1
}

\author{
Vincent Chevrier, ${ }^{1}$ Derek W. G. Sears, ${ }^{1,2}$ Julie D. Chittenden, ${ }^{1,2}$ Larry A. Roe, ${ }^{1,3}$ \\ Richard Ulrich, ${ }^{1,4}$ Kathryn Bryson, ${ }^{1}$ Lisa Billingsley, ${ }^{1}$ and Jennifer Hanley ${ }^{5}$ \\ Received 10 October 2006; revised 4 December 2006; accepted 7 December 2006; published 18 January 2007.
}

[1] We have studied the sublimation of ice buried beneath $\leq 200 \mathrm{~mm}$ of JSC Mars-1 model regolith under simulated Mars conditions. As expected, even thin layers of regolith cause large decreases in sublimation rate, up to one order of magnitude at $50 \mathrm{~mm}$. When the depth of the regolith was 50 to $200 \mathrm{~mm}$ we detected water desorbing from the overlying layers for which we were able to determine a desorption coefficient of $1.45 \pm 0.5 \times 10^{-3} \mathrm{~h}^{-1}$. After allowing for the effect of desorption, we found that the diffusion coefficient for water vapor through our regolith is $1.74 \pm 0.70 \times 10^{-4} \mathrm{~m}^{2} \mathrm{~s}^{-1}$, in excellent agreement with theoretical values. We thus find that a $1-\mathrm{m}$ thick layer of ice buried below a meter of regolith resembling JSC Mars-1 on Mars at $235 \mathrm{~K}$ would last $\sim 800$ years. Citation: Chevrier, V., D. W. G. Sears, J. D. Chittenden, L. A. Roe, R. Ulrich, K. Bryson, L. Billingsley, and J. Hanley (2007), Sublimation rate of ice under simulated Mars conditions and the effect of layers of mock regolith JSC Mars-1, Geophys. Res. Lett., 34, L02203, doi:10.1029/2006GL028401.

\section{Introduction}

[2] Ice has been detected over most of Mars. Except for the polar caps, the ice is covered by layers of regolith [Boynton et al., 2002; Feldman et al., 2004; Mellon et al., 2004]. These regolith layers are crucial in protecting and stabilizing ice primarily by providing a barrier to sublimation [Farmer, 1976; Jakosky, 1985]. Under appropriate conditions, such stabilization could eventually cause a pathway to the formation of liquid water [Hecht, 2002]. Detailed consideration of diffusion and adsorption of water vapor in the martian regolith has lead to the suggestion that the regolith could be an important reservoir of water on Mars [Farmer, 1976; Jakosky, 1985; Schorghofer and Aharonson, 2005; Smoluchowski, 1968; Zent and Quinn, 1997], and this has important consequences for climate and water vapor circulation models [Haberle et al., 2001; Jakosky et al., 1997; Mellon et al., 2004; Schorghofer and Aharonson, 2005]. However, details of this process and

\footnotetext{
${ }^{1}$ W. M. Keck Laboratory for Space Simulations, Arkansas Center for Space and Planetary Sciences, University of Arkansas, Fayetteville, Arkansas, USA.

${ }^{2}$ Also at Department of Chemistry and Biochemistry, University of Arkansas, Fayetteville, Arkansas, USA

${ }^{3}$ Also at Department of Mechanical Engineering, University of Arkansas, Fayetteville, Arkansas, USA.

${ }^{4}$ Also at Department of Chemical Engineering, University of Arkansas, Fayetteville, Arkansas, USA.

${ }^{5}$ Department of Earth and Atmospheric Sciences, Cornell University, Ithaca, New York, USA.
}

Copyright 2007 by the American Geophysical Union. 0094-8276/07/2006GL028401\$05.00 the thickness of regolith necessary to stabilize the ice are poorly known, mainly because of the lack of quantitative parameters [Haberle et al., 2001; Schorghofer and Aharonson, 2005]. Indeed most of the values for diffusion coefficient of water vapor in martian regolith used in models are theoretical estimates that lack experimental verification [Farmer and Doms, 1979; Smoluchowski, 1968]. The purpose of this study is to investigate the extent to which dust layers on an ice surface suppress evaporation and thereby determine experimentally the diffusion coefficient of water vapor in the regolith under Mars conditions.

\section{Experimental}

[3] The apparatus used in the present study is a slightly modified version of the equipment used by Sears and Chittenden [2005] and Sears and Moore [2005] for their studies of the evaporation of water and brine on Mars (Figure 1). It consists of a stainless steel vacuum chamber with a volume of $0.6 \mathrm{~m}^{3}$ that can be maintained at temperatures between $-20^{\circ} \mathrm{C}$ and $0^{\circ} \mathrm{C}$ by a methanol/dry-ice slurry circulating around the chamber. Conditions in the chamber are monitored by eight thermocouples, two pressure gauges, two hygrometers and closed circuit television. For the present measurements, the chamber was evacuated to $<0.1$ mbar and backfilled to $1 \mathrm{~atm}$ with dry gaseous $\mathrm{CO}_{2}$. Graduated cylinders $(100 \mathrm{~mL})$ containing ice-regolith columns, which had been kept in a freezer overnight, were then placed in the chamber on a top-loading balance with a thermocouple on the soil surface (Figure 1). The mock regolith used for these studies, JSC Mars-1 regolith [Allen et al., 1998], is a palagonitic soil with an initial volatile content of $6.65 \pm 0.24 \mathrm{wt} \%$ (based on the average mass loss of 20 samples heated at $110^{\circ} \mathrm{C}$ for 15 days). The chamber was then evacuated to $7 \mathrm{mbar}(700 \mathrm{~Pa})$ and allowed to settle down for 20 minutes, at which point the mass, pressure, temperatures (of the atmosphere, walls, sample and coolant), and humidity were recorded every 2 to 5 minutes for 1 to 2 hours. The total pressure was maintained between 6.5 and 8.0 mbar, and the temperature was maintained constant by adding the required amounts of dry ice to the methanol coolant. Dry $\mathrm{CO}_{2}$ was introduced continuously to maintain an average humidity of $6.1 \pm 0.9 \%$, which corresponds to a partial pressure of water of $\sim 0.37$ mbar. A total of 140 experiments were performed, 5-15 for each of ten depths of regolith.

\section{Results}

[4] The mass versus time curve for each sample was highly linear with $\mathrm{R}^{2}$ better than 0.99 (Figure 2). This was 


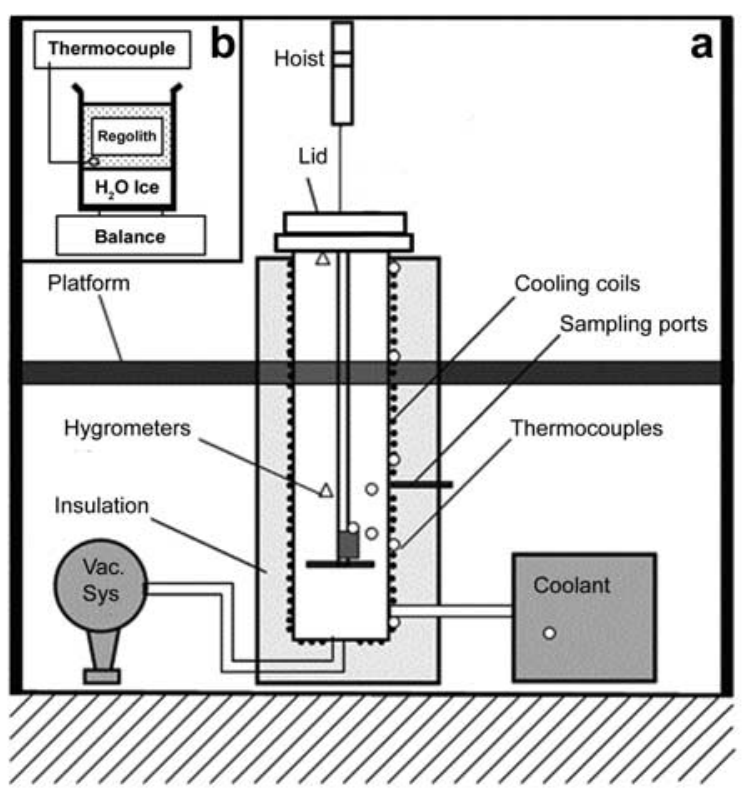

Figure 1. (a) Schematic diagram of the simulation chamber and (b) experimental set-up for the present experiments. The $61 \mathrm{~cm}$ diameter and $208 \mathrm{~cm}$ deep chamber is located in a specially constructed room with an adjacent independent control room. The chamber is equipped with cooling system, vacuum system, thermocouples (shown as open circles), hygrometers (shown as triangles). Inset shows the experimental set-up in which ice is allowed to sublime through a simulated regolith of JSC Mars-1 which sits on a top loading balance. A thermocouple is placed at the interface between the ice and the regolith to monitor the temperature of the sample.

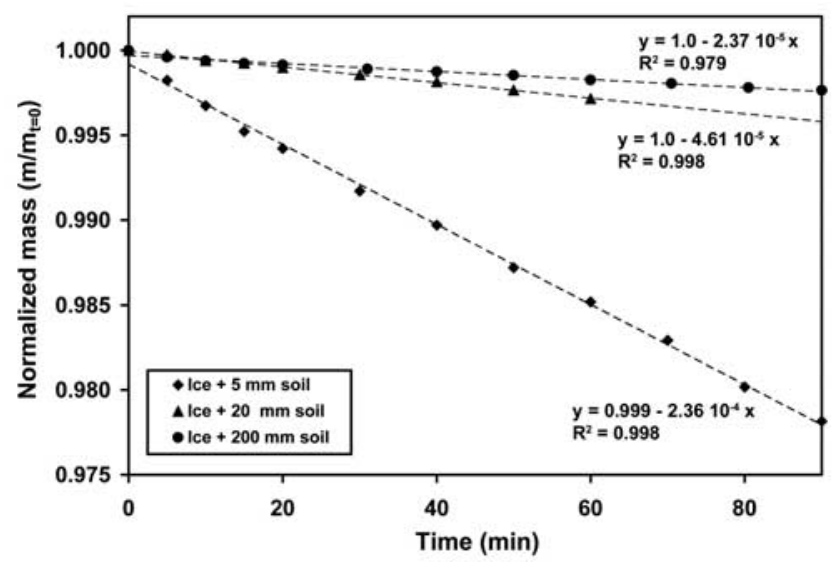

Figure 2. Examples of our experimental data. Fractional mass loss is plotted against time. The experimental uncertainties are comparable to or smaller than the size of the symbols. The lines through the data are regression lines and the equation and $\mathrm{R}^{2}$ value are indicated. The linearity of these trends indicates that we have achieved steady state in our experiments.

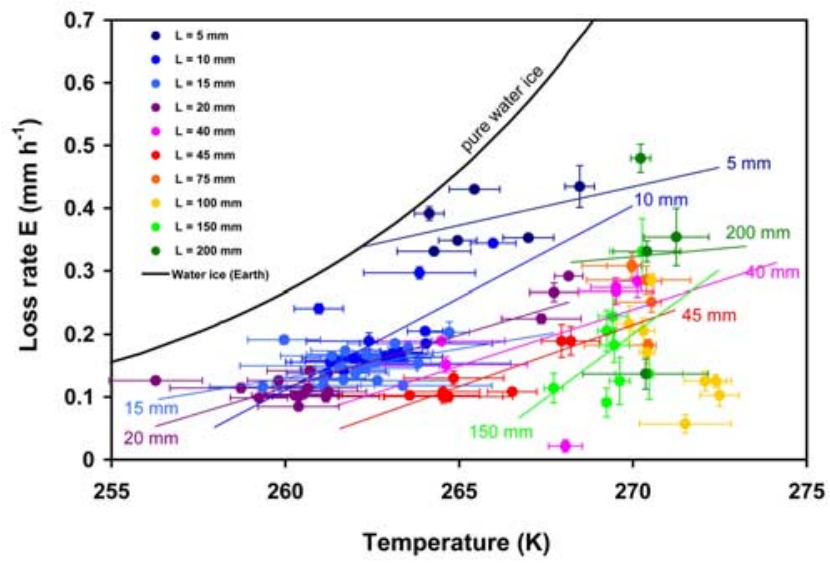

Figure 3. Rate of mass loss for water ice (converted from $\mathrm{g} \mathrm{min}^{-1}$ to $\mathrm{mm} \mathrm{h}^{-1}$ ) as a function of temperature for various thicknesses of JSC Mars-1 martian soil simulant. Sublimation of pure ice as determined from the Ingersoll equation [Ingersoll, 1970; Sears and Moore, 2005] is also shown for the Earth (thick black line). The thin colored lines correspond to the regression line through each set of data.

used to obtain the total mass loss rate in $\mathrm{g} \mathrm{min}^{-1}$, which was converted into $\mathrm{mm} \mathrm{h}^{-1}$ by dividing by the water ice density and the surface area of the sample (Figure 3). The mass loss rate varies from 0.02 to $0.45 \mathrm{~mm} \mathrm{~h}^{-1}$ over a temperature range of 257 to $273 \mathrm{~K}$. Linear regression lines through the data at each burial depth have been added for clarity in Figure 3, but there is no implication that these trends are truly linear. The relative standard error on the determination of individual mass loss rate was generally $<5 \%$, but the data in Figure 3 show greater than 5\% scatter (especially at greater depths) because of differences in operating conditions, mainly variations in temperature, porosity, compaction and uniformity of the regolith.

\section{Discussion}

\subsection{Comparison of the Observed Sublimation Rates} With Those of Pure Water Ice

[5] The curve for pure water ice sublimation rate (no regolith covering), calculated from the expression:

$$
E_{S}=0.612 \Delta \eta \rho_{a t m} D\left[\frac{\frac{\Delta \rho}{\rho} g}{\nu^{2}}\right]^{\frac{1}{3}}
$$

is also shown in Figure 3. This relationship was described by Ingersoll [1970] and confirmed experimentally in our previous papers [Moore and Sears, 2006; Sears and Chittenden, 2005; Sears and Moore, 2005]. In this expression, $\mathrm{E}_{\mathrm{S}}$ is the sublimation rate in $\mathrm{mm} \mathrm{h}^{-1}, \Delta \eta$ is the concentration difference at the surface of the sample and at distance, $\rho_{\mathrm{atm}}$ is the atmospheric density, $\mathrm{D}$ is the diffusion coefficient for water in $\mathrm{CO}_{2}\left(1.4 \times 10^{-3} \mathrm{~m}^{2} \mathrm{~s}^{-1}\right), \nu$ is the kinematic viscosity of $\mathrm{CO}_{2}\left(6.93 \times 10^{-4} \mathrm{~m}^{2} \mathrm{~s}^{-1}\right)$, and $\mathrm{g}$ is acceleration due to gravity $\left(\mathrm{m} \mathrm{s}^{-2}\right)$. The term $\Delta \eta$ refers to the water vapor concentration difference between the 

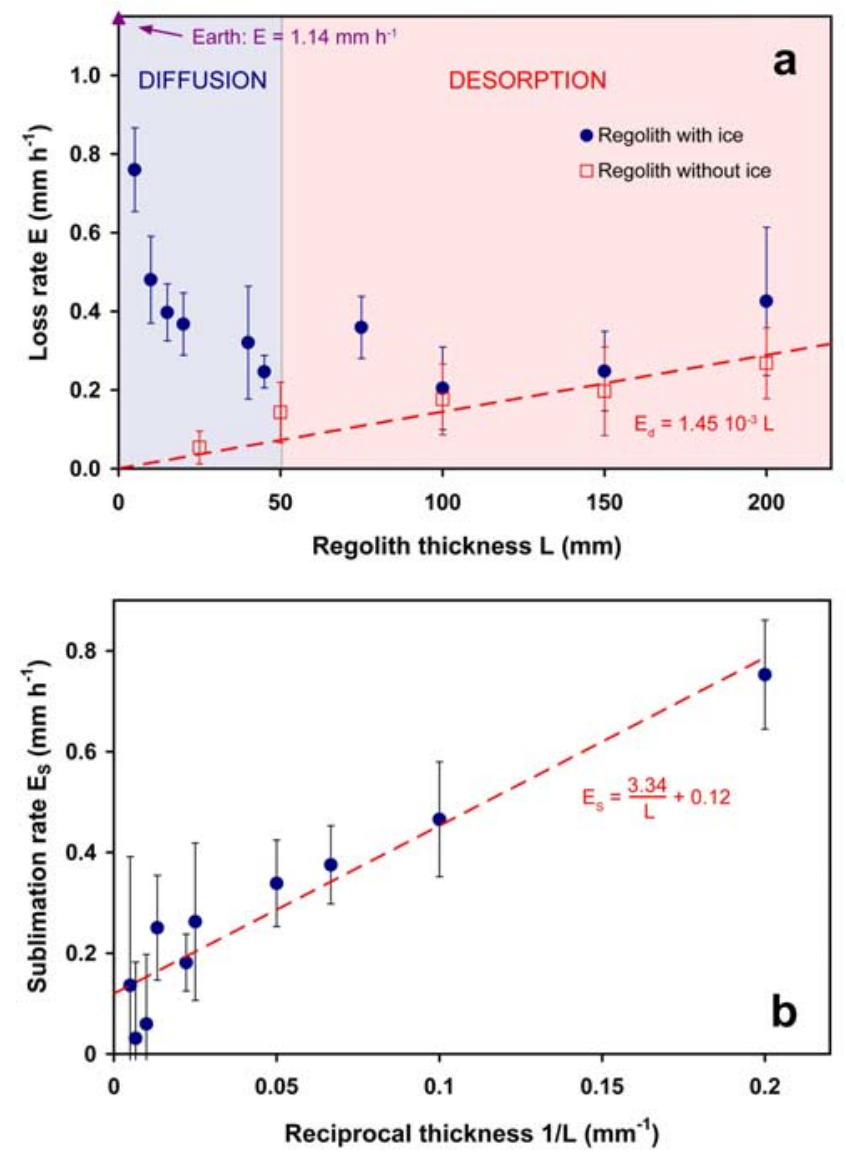

Figure 4. (a) Total mass loss rate of water (filled symbols) and desorption rate of water vapor from the regolith (open symbols) as a function of the thickness of the regolith layer. Mass loss rates $\left(\mathrm{g} \mathrm{min} \mathrm{m}^{-1}\right)$ have been converted into the equivalent water ice thickness $\left(\mathrm{mm} \mathrm{h}^{-1}\right)$. A regression line has been drawn through the desorption data. The closed triangle represents the evaporation of pure water ice (i.e. without regolith) as determined experimentally [Sears and Moore, 2005] and by using the Ingersoll equation [Ingersoll, 1970]. For regolith thicknesses less than about $50 \mathrm{~mm}$ (blue zone), mass loss is governed mainly by the diffusion process, but at greater thicknesses (red zone) desorption accounts for the main release of water from the regolith used in these simulation experiments. (b) Sublimation rate of ice (corrected for the desorption of the regolith) as a function of reciprocal regolith depth $(1 / \mathrm{L})$. The sublimation rate for pure ice is also indicated. The regression line through the data indicates that the sublimation of ice through a regolith layer follows the relationship $\mathrm{E}=3.34 / \mathrm{L}+0.12$, suggesting a diffusion controlled process.

surface and the atmosphere and $\Delta \rho / \rho$ corresponds to the relative density difference between the surface and the atmosphere. The estimation of these terms has been discussed previously [Ingersoll, 1970; Sears and Chittenden, 2005; Sears and Moore, 2005]. The values plotted in Figure 3, and the theory line, are applicable to Earth's gravity and may be corrected for the lower gravity on Mars by multiplying by 0.726 [Sears and Chittenden, 2005; Sears and Moore, 2005]. As expected, the regolith layers are attenuating the mass loss rate (by factors of up to about 7 or 8 ), and the rate of mass loss is positively related to temperature (except for data obtained at 75 and $100 \mathrm{~mm}$ depths where the temperature spread is too small to see these effects).

\subsection{Converting the Data to a Common Temperature and Pressure}

[6] The highly linear nature of our data (see Figure 2) indicates that our experiments are achieving steady state conditions. Furthermore, as we suppose that the mass loss for our columns results mostly from sublimation at the interface between the ice and the regolith, we can correct each data point from its temperature and humidity to $273 \mathrm{~K}$ and $10 \mathrm{~Pa}$ of atmospheric $\mathrm{H}_{2} \mathrm{O}$. Then we can study the transport properties of the regolith without the effect of the temperature and humidity. The treatment of Farmer [1976] can be combined with the ideal gas law to account for the differences in temperature and atmospheric pressure:

$$
E_{S}=\frac{D M_{H_{2} O} p_{\text {sat }}}{L R T_{S} \mu_{\text {ice }}}\left[1-\frac{T_{S} p_{\text {atm }}}{T_{\text {atm }} p_{\text {sat }}}\right]
$$

where $E_{S}$ is the sublimation rate, $D$ is the diffusion coefficient in the regolith, $\mathrm{L}$ is the regolith thickness, $\mathrm{T}_{\mathrm{S}}$ is the temperature of the surface, $\mathrm{T}_{\mathrm{atm}}$ is the temperature of the atmosphere, $p_{a t m}$ is the partial pressure of water in the atmosphere, $\mu_{\text {ice }}$ is the density of the ice $\left(917 \mathrm{~kg} \mathrm{~m}^{-3}\right)$ and $\mathrm{R}$ is the gas constant. For depths below $\sim 100 \mathrm{~mm}$, the average mass loss rate decreases as expected with the soil thickness to about $0.20 \pm 0.1 \mathrm{~mm} \mathrm{~h}^{-1}$ at $100 \mathrm{~mm}$, but contrary to expectations at greater depths the mass loss rate increases with depth up to $0.43 \pm 0.19 \mathrm{~mm} \mathrm{~h}^{-1}$ at $200 \mathrm{~mm}$ (Figure 4a). Such result suggests that sublimation is not the only phenomenon responsible for mass loss for the ice/ regolith samples, but that a secondary contribution - that increases with soil thickness - is responsible for the mass loss at higher depth. We suggest that this second process is desorption which we discuss in the next section.

\subsection{Desorption Effects}

[7] Desorption of water by Mars regolith simulants has been studied by Zent and his colleagues [Zent et al., 2001; Zent and Quinn, 1995, 1997]. To confirm and quantify this process, we performed a series of measurements in which we placed various thicknesses of regolith in the chamber without an underlying ice layer. The measured desorption rate $\left(\mathrm{g} \mathrm{min}^{-1}\right)$ was converted into an "ice-loss equivalent" $\left(\mathrm{mm} \mathrm{h}^{-1}\right)$ in order to facilitate comparison with our sublimation data. The results are shown in Figure 4a. The rate of water desorbed from the regolith layer increased linearly with regolith thickness, from $5.4 \pm 4.2 \times 10^{-2} \mathrm{~mm} \cdot \mathrm{h}^{-1}$ at $25 \mathrm{~mm}$ regolith thickness to $0.27 \pm 0.09 \mathrm{~mm} \mathrm{~h}^{-1}$ at $200 \mathrm{~mm}$ regolith thickness. For reasonable values of diffusion coefficient between $10^{-3}$ and $10^{-5} \mathrm{~m}^{2} \mathrm{~s}^{-1}$ [Smoluchowski, 1968 , and $20 \mathrm{~cm}$ of regolith, we calculate that steady-state is reached in 1 to 15 minutes. Thus, in these conditions all the water desorbed is immediately evacuated from the regolith, explaining the linearity of the desorption line in Figure $4 \mathrm{a}$. This linear dependence of the desorption rate with soil thickness also suggests that, to a reasonable approxi- 
mation, the Langmuir desorption theory is applicable [Zent et al., 2001]:

$$
\mathrm{E}_{\mathrm{d}}=\mathrm{k}_{\mathrm{d}} \mathrm{L}
$$

with $\mathrm{E}_{\mathrm{d}}$ being the desorption rate (converted from $\mathrm{g} \min ^{-1}$ to $\mathrm{mm} \mathrm{h}^{-1}$ ), $\mathrm{k}_{\mathrm{d}}$ the desorption coefficient, and $\mathrm{L}$ the soil thickness. A linear fit to the present data gives a desorption coefficient $\mathrm{k}_{\mathrm{d}}=1.45 \pm 0.5 \times 10^{-3} \mathrm{~h}^{-1}$. The desorption data and mass loss data converge at depths greater than $\sim 50 \mathrm{~mm}$, suggesting that for regolith depths less than $\sim 50 \mathrm{~mm}$ sublimation is the main process while at depths greater than $50 \mathrm{~mm}$ desorption is the dominant process in releasing water into the atmosphere of the chamber.

\subsection{Diffusion Effects}

[8] In Figure $4 \mathrm{~b}$ we compare the predictions of equation (2) with the present experimental data. The present data form a linear array on a plot of $E_{S}$ against $1 / L$, confirming that we have a steady state process described by equation (3). The slope of a line through the data indicates water vapor diffusing through JSC Mars-1 regolith simulant with a diffusion coefficient of $1.74 \pm 0.70 \times 10^{-4} \mathrm{~m}^{2} \mathrm{~s}^{-1}$. This result is in agreement with the usual theoretical estimation for the diffusion of water vapor through the martian regolith of $\sim 10^{-4} \mathrm{~m}^{2} \mathrm{~s}^{-1}$ [Schorghofer and Aharonson, 2005; Smoluchowski, 1968]. The residual sublimation rate of $0.12 \pm 0.05 \mathrm{~mm} . \mathrm{h}^{-1}$ for an infinite regolith layer (the $y$ - intercept on Figure $4 b$ ) is the result of experimental uncertainties and possible edge effects in our experiment. While 0.12 is essentially equal to the $2 \sigma$ uncertainty on an individual data point, we believe some of the offset is due to a change in sublimation regime from diffusion through a soil (as described by the modified Farmer equation (2)) to diffusion through the atmosphere (as described by the Ingersoll equation (1)).

[9] With the value of the diffusion coefficient, we can estimate the time $t$ to reach steady-state, at which the Farmer model can be applied, using the equation:

$$
t=\frac{L^{2}}{4 D}
$$

For our maximum mock regolith thickness, i.e $20 \mathrm{~cm}$, the calculated time is about $60 \mathrm{~s}$, a value very much below the 20 min settling down time allowed for in our experiments.

\subsection{Dependence of the Diffusion Coefficient on Temperature}

[10] We have not been able to observe any statistical variation of the diffusion coefficient with temperature (recalculated from each data point using equation (2) before temperature/humidity correction). We suspect that this is mostly because the temperature range of our data is very limited in relation to the scatter in the data, but we can conclude that our data show no evidence for a very marked dependence of the diffusion coefficient on temperature at these temperatures.

\subsection{Significance for the Stability and Survival Times of Subsurface Ice}

[11] The diffusion coefficient we have determined indicates that the diffusion of water vapor and the response of the regolith to any change in atmospheric humidity will be relatively fast. However, the evaporation rate is also strongly dependent on the temperature of the ice surface (viz., equation (3)). Knowing the diffusion coefficient in the regolith, we can use equation (3) to determine the survival time of a layer of ice under a regolith layer. A $1 \mathrm{~m}$-thick layer of ice buried under $\sim 1 \mathrm{~m}$ of regolith as suggested by the Odyssey neutron and gamma spectrometer data [Jakosky et al., 2005] and discussed by earlier workers [Farmer and Doms, 1979; Paige, 1992; Schorghofer and Aharonson, 2005], would last $\sim 800$ years at the maximal martian temperature of $235 \mathrm{~K}$ consistent with models for the metastability of ice in the equatorial regions [Jakosky et al., 2005]. Moreover, any temperature below $160-170 \mathrm{~K}$ will generate saturation pressures on the surface well below the atmospheric water humidity between 0.001 and 1 [Zent et al., 2001], preventing any sublimation of ice. Such understanding and quantification of the interactions between subsurface ice and the surrounding regolith is important to interpreting data to be obtained by the upcoming Phoenix mission [Smith, 2006].

[12] Acknowledgments. We are grateful to Walter Graupner for laboratory assistance. A major award from the W.M. Keck Foundation, Los Angeles, supports the laboratory and the present work.

\section{References}

Allen, C. C., R. V. Morris, K. M. Jager, D. C. Golden, D. J. Lindstrom, M. M. Lindstrom, and J. P. Lockwood (1998), Martian regolith simulant JSC Mars-1, Lunar Planet. Sci., XXIX, abstract 1690.

Boynton, W. V., et al. (2002), Distribution of hydrogen in the near surface of Mars: Evidence for subsurface ice deposits, Science, 297, $81-85$.

Farmer, C. B. (1976), Liquid water on Mars, Icarus, 28, 279-289.

Farmer, C. B., and P. E. Doms (1979), Global seasonal variations of water vapor on Mars and the implications for permafrost, J. Geophys. Res., 84, $2881-2888$.

Feldman, W. C., et al. (2004), Global distribution of near-surface hydrogen on Mars, J. Geophys. Res., 109, E09006, doi:10.1029/2003JE002160.

Haberle, R. M., C. P. McKay, J. Schaeffer, N. A. Cabrol, E. A. Grin, A. P. Zent, and R. Quinn (2001), On the possibility of liquid water on presentday Mars, J. Geophys. Res., 106(E10), 23,317-23,326.

Hecht, M. H. (2002), Metastability of liquid water on Mars, Icarus, 156, $373-386$.

Ingersoll, A. P. (1970), Mars: Occurrence of liquid water, Science, 168(3934), 972-973.

Jakosky, B. M. (1985), The seasonal cycle of water on Mars, Space Sci. Rev., 41(1-2), 131-200.

Jakosky, B. M., A. P. Zent, and R. W. Zurek (1997), The Mars water cycle: Determining the role of exchange with the regolith, Icarus, 130, $87-95$.

Jakosky, B. M., M. T. Mellon, E. S. Varnes, W. C. Feldman, W. V. Boynton, and R. M. Haberle (2005), Mars low-latitude neutron distribution: Possible remnant near-surface water ice and a mechanism for its recent emplacement, Icarus, 175, 58-67.

Mellon, M. T., W. C. Feldman, and T. H. Prettyman (2004), The presence and stability of ground ice in the southern hemisphere of Mars, Icarus, 169, 240-324.

Moore, S. R., and D. W. G. Sears (2006), On laboratory simulation and the effect of small temperature oscillations about the freezing point and ice formation on the evaporation rate of water on Mars, Astrobiology, 6(4), $644-650$

Paige, D. A. (1992), The thermal stability of near-surface ground ice on Mars, Nature, 356, 43-45.

Schorghofer, N., and O. Aharonson (2005), Stability and exchange of subsurface ice on Mars, J. Geophys. Res., 110, E05003, doi:10.1029/ 2004JE002350.

Sears, D. W. G., and J. D. Chittenden (2005), On laboratory simulation and the temperature dependence of the evaporation rate of brine on Mars, Geophys. Res. Lett., 32, L23203, doi:10.1029/2005GL024154. 
Sears, D. W. G., and S. R. Moore (2005), On laboratory simulation and the evaporation rate of water on Mars, Geophys. Res. Lett., 32, L16202, doi:10.1029/2005GL023443.

Smith, P. H. (2006), Science considerations driving the choice of the Phoenix mission landing site, Lunar Planet. Sci., XXXVII, abstract 1910.

Smoluchowski, R. (1968), Mars: Retention of ice, Science, 159(3821), $1348-1350$

Zent, A. P., and R. C. Quinn (1995), Simultaneous adsorption of $\mathrm{CO}_{2}$ and $\mathrm{H}_{2} \mathrm{O}$ under Mars-like conditions and application to the evolution of the Martian climate, J. Geophys. Res., 100(E3), 5341-5349.

Zent, A. P., and R. C. Quinn (1997), Measurement of $\mathrm{H}_{2} \mathrm{O}$ under Mars-like conditions: effects of adsorbent heterogeneity, J. Geophys. Res., 102(E4), 9085-9095
Zent, A. P., D. J. Howard, and R. C. Quinn (2001), $\mathrm{H}_{2} \mathrm{O}$ adsorption on smectites: Application to the diurnal variation of $\mathrm{H}_{2} \mathrm{O}$ in the Martian atmosphere, J. Geophys. Res., 106(E7), 14,667-14,674.

L. Billingsley, K. Bryson, V. Chevrier, J. D. Chittenden, L. A. Roe, D. W. G. Sears, and R. Ulrich, W. M. Keck Laboratory for Space Simulations, Arkansas Center for Space and Planetary Sciences, University of Arkansas, Fayetteville, AR 72701, USA. (vchevrie@uark.edu)

J. Hanley, Department of Earth and Atmospheric Sciences, Cornell University, Ithaca, NY 14853, USA. 